\title{
EUCLIDEAN SHIFT-TWIST SYMMETRY IN POPULATION MODELS OF SELF-ALIGNING OBJECTS*
}

\author{
PAUL C. BRESSLOFF ${ }^{\dagger}$
}

\begin{abstract}
We consider the symmetry properties of a general class of nonlocal population models describing the aggregation and alignment of oriented objects in two dimensions. Such objects could be at the level of molecules, cells, or whole organisms. We show that the underlying interaction kernel is invariant under the so-called shift-twist action of the Euclidean group acting on the space $\mathbf{R}^{2} \times S^{1}$. This group action was previously studied within the context of a continuum model of primary visual cortex. We use perturbation methods to solve the eigenvalue problem arising from linearization about a homogeneous state, and then use equivariant bifurcation theory to identify the various types of doubly periodic patterns that are expected to arise when the homogeneous state becomes unstable. We thus establish that two distinct forms of spatio-angular order can occur, corresponding to scalar and pseudoscalar representations of the Euclidean group.
\end{abstract}

Key words. cell alignment, actin cytoskeleton, animal aggregation, Euclidean symmetry, selforganization, population models, integro-differential equations

AMS subject classifications. 92B05, 37G40

DOI. $10.1137 / \mathrm{S} 0036139903436017$

1. Introduction. A wide variety of self-organizing biological systems exhibit aggregation and alignment phenomena. These occur spontaneously due to mutual interactions between the individual elements of a population, in which both the relative position and the relative orientation of the individuals have a significant effect on the nature of the interactions. The underlying population can consist of molecules, cells, or whole organisms. A well-known example of the last category is the aggregation of animal herds, fish schools, and flocks of birds, in which the members of the group tend to align their bodies with each other and move in a common direction [14]. Such behavior provides a defense against predators. Examples at the cellular and molecular levels are the alignment of mammalian fibroblast cells within densely formed patches [10] and the alignment of actin filaments forming a scaffolding structure within a cell [22]. In order to investigate the important role of alignment in population survival and in the properties of biological materials, a number of continuum models of interacting oriented objects have been developed, with applications to animal social groups $[19,14,6]$, fibroblasts $[16,17,8]$, and actin [7, 12, 21]. All of these models are formulated in terms of integro-differential equations describing the evolution of the distribution of oriented elements in space. It is typically assumed that the interaction terms involve convolutions of the population distribution with some linear kernel. Convolutions with respect to orientation are natural, since an individual can interact with a neighboring cell having any relative orientation, whereas convolutions with respect to spatial position can be justified by assuming that signaling between individuals happens on a much faster time-scale than aggregation and alignment [15].

In this paper we investigate how symmetries of the interaction kernel determine the types of spatio-angular patterns that can emerge through a Turing-like instability of a homogeneous state. For concreteness, we focus on a diffusion-advection equation

\footnotetext{
*Received by the editors October 6, 2003; accepted for publication (in revised form) January 5, 2004; published electronically June 22, 2004.

http://www.siam.org/journals/siap/64-5/43601.html

${ }^{\dagger}$ Department of Mathematics, University of Utah, 155 South 1400 East, Salt Lake City, UT 84112 (bressloff@math.utah.edu).
} 
for a population of oriented objects distributed in the two-dimensional plane. This corresponds to the second of three classes of model previously studied by Mogilner and Edelstein-Keshet [18], in which nonlocal interactions induce a rotation about the center of mass of each element as well as a linear drift of the center of mass in the plane. (The other two models are less realistic in the sense that they treat the alignment process as instantaneous, although they do exhibit the same qualitative behavior since they have the same underlying symmetries.) Mogilner and EdelsteinKeshet [18] solved the eigenvalue problem arising from a linear stability analysis of the homogeneous state in the case of a separable interaction kernel, that is, one in which variations in the strength of interaction with respect to relative orientation and relative position are uncorrelated. Such a kernel is invariant under the action of the product group $\mathbf{E}(2) \times \mathbf{O}(2)$, where $\mathbf{E}(2)$ denotes the Euclidean group of rigid body motions in the plane $\mathbf{R}^{2}$ and $\mathbf{O}(2)$ consists of rotations and reflection on the circle $S^{1}$. However, it is often found that individuals with similar orientations have stronger interactions when they are collinear in the plane, implying that the interaction kernel is nonseparable [6]. (A specific example of a nonseparable kernel was briefly considered in [18], but general symmetry properties were not addressed.) Here we show that these more realistic kernels are invariant with respect to the so-called shift-twist action of $\mathbf{E}(2)$ acting on the space $\mathbf{R}^{2} \times S^{1}$, and we explore the consequences of this symmetry for pattern formation. Note that the same group action has recently been analyzed within the context of continuum models of the visual cortex, where the nonlocal interactions are mediated by axonal connections between neurons that are tuned to respond to oriented visual stimuli $[3,4]$. Shift-twist invariant kernels also play a central role in a recently proposed computational algorithm for grouping and joining edges that form the boundaries of objects in a visual image [24].

We begin by describing the nonlocal population model for the aggregation and alignment of oriented objects in two dimensions and discussing its symmetry properties (section 2). We show that the resulting diffusion-advection equation is equivariant with respect to the shift-twist action of the Euclidean group due to the invariance of the underlying interaction kernel. We then use perturbation methods to solve the eigenvalue problem arising from linearization about a homogeneous state and determine marginal stability conditions (section 3). Finally, we use equivariant bifurcation theory to identify the various types of doubly periodic patterns that are expected to arise when the homogeneous state becomes unstable, and thus establish that two distinct forms of spatio-angular order can occur, corresponding to the so-called scalar and pseudoscalar representations of the Euclidean group (section 4). Interestingly, analogous results [5] have been obtained for the Landau-de Gennes model of a nematic liquid crystal [9], where the oriented objects are rod-like molecules that interact by electrostatic attraction or repulsion.

2. Description of the model and its symmetries. Let $f(\mathbf{r}, \theta, t)$ denote the distribution of oriented objects in a two-dimensional domain $\mathcal{D} \subset \mathbf{R}^{2}$ at time $t$ with $\mathbf{r} \in \mathcal{D}$ and $-\pi<\theta \leq \pi$. It is assumed that the total number of objects $N$ is conserved; that is, $\dot{N}=0$ with

$$
N=\int_{\mathcal{D}} \int_{-\pi}^{\pi} f(\mathbf{r}, \theta, t) \frac{d \theta}{2 \pi} d^{2} \mathbf{r}
$$

In cases where the population grows (due to cell proliferation, for example), $N$ may still be treated as a constant, provided that the growth process is adiabatic. The pop- 
ulation distribution $f$ is taken to evolve according to the diffusion-advection equation

$$
\frac{\partial f}{\partial t}=D_{1} \frac{\partial^{2} f}{\partial \theta^{2}}+D_{2} \nabla^{2} f-\frac{\partial J_{\theta}}{\partial \theta}-\nabla \cdot \mathbf{J}_{r} .
$$

Here $D_{1}$ and $D_{2}$ are angular and spatial diffusion constants, $J_{\theta}$ is the flux arising from changes in the orientation of objects, and $\mathbf{J}_{r}$ is the flux arising from motion in the plane:

$$
J_{\theta}=f \frac{d \theta}{d t}, \quad \mathbf{J}=f \frac{d \mathbf{r}}{d t} .
$$

Following Mogilner and Edelstein-Keshet [16, 18], we assume that inertial forces can be neglected so that the velocities are simply proportional to the driving forces,

$$
\frac{d \theta}{d t}=\eta_{1} F_{\theta}, \quad \frac{d \mathbf{r}}{d t}=\eta_{2} \mathbf{F}_{r} .
$$

The forces are taken to be conservative; that is, each can be expressed in terms of the gradient of an underlying potential function $V$ :

$$
F_{\theta}=\frac{\partial V}{\partial \theta}, \quad \mathbf{F}_{r}=\nabla V
$$

where $V$ is given by the integral of $f$ with respect to a linear kernel $W$,

$$
V(\mathbf{r}, \theta)=W * f(\mathbf{r}, \theta) \equiv \int_{\mathcal{D}} \int_{-\pi}^{\pi} W\left(\mathbf{r}, \theta \mid \mathbf{r}^{\prime}, \theta^{\prime}\right) f\left(\mathbf{r}^{\prime}, \theta^{\prime}\right) \frac{d \theta}{2 \pi} d^{2} \mathbf{r} .
$$

(One could also consider a more general situation in which angular and planar motion are generated by two distinct potentials [18].) Substituting (2.3), (2.4), (2.5), and (2.6) into (2.2) leads to the following model equation:

$$
\frac{\partial f}{\partial t}=D_{1} \frac{\partial^{2} f}{\partial \theta^{2}}+D_{2} \nabla^{2} f-\eta_{1} \frac{\partial}{\partial \theta}\left(f \frac{\partial(W * f)}{\partial \theta}\right)-\eta_{2} \nabla \cdot(f \nabla(W * f)) .
$$

2.1. The interaction kernel. We now specify the form of the interaction kernel $W$. Consider a local patch of individuals at point $\mathbf{r}$ with orientation $\theta$. These will move in the plane and reorient as a result of the influence from other patches at $\mathbf{r}^{\prime}$ with orientation $\theta^{\prime}$ (see Figure 2.1(a)). It is likely that the interactions depend on three distinct factors [6, 18]: (i) the Euclidean distance $\left|\mathbf{r}-\mathbf{r}^{\prime}\right|$, (ii) the relative orientation $\theta-\theta^{\prime}$, and (iii) the relative alignment in the plane $\psi=\arg \left(\mathbf{r}-\mathbf{r}^{\prime}\right)-\theta$. One way to understand the third factor is to consider the case of parallel objects that are equidistant in the plane but are either collinear or flanking each other (see Figure 2.1(b)). Given the fact that each object is elongated, it is possible that collinear objects tend to influence each other more strongly than flanking objects. In the cellular or molecular case this would arise due to differences in the contact areas of the individuals, whereas in animal social groups this would reflect differences in the ability to sense individuals in different relative directions. In the latter case, the influence of individuals behind a given animal would also tend to be weaker than on the sides or front. If we assume that the three effects are independent, then the interaction kernel can be decomposed into the product form (see [6])

$$
W\left(\mathbf{r}, \theta \mid \mathbf{r}^{\prime}, \theta^{\prime}\right)=G\left(\left|\mathbf{r}-\mathbf{r}^{\prime}\right|\right) H\left(\theta-\theta^{\prime}\right) \Delta\left(\arg \left(\mathbf{r}-\mathbf{r}^{\prime}\right)-\theta\right) .
$$




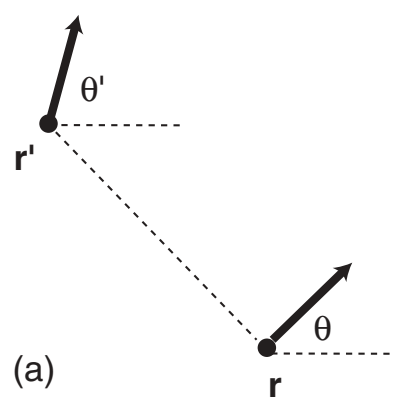

FIG. 2.1. (a) Two oriented objects in the plane. (black) and flanking (gray) oriented objects.

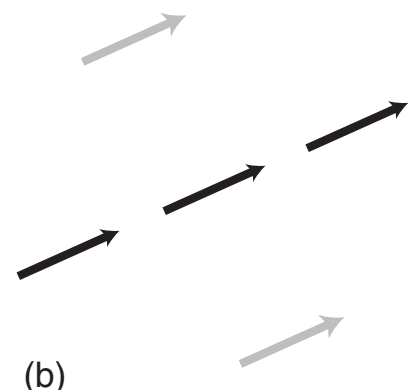

(b) Difference in the influence of collinear

We now discuss each of the terms appearing in (2.8).

The strength of interactions decreases as a function of spatial separation so that $G(r)$ is a monotonically decreasing function of $r$. For concreteness, we take $G$ to be a Gaussian

$$
G(r)=\frac{1}{2 \pi \sigma^{2}} \mathrm{e}^{-r^{2} / 2 \sigma^{2}},
$$

where $\sigma$ denotes the effective range of interactions. We fix the spatial scale by setting $\sigma=1$. Throughout this paper we assume that the range of interactions is at least a few orders of magnitude smaller than the size of the domain $\mathcal{D}$. This allows us to treat the spatial domain as infinite so that we can ignore boundary effects. The angular contribution $H(\theta)$ is assumed to be an even function of $\theta$ with one or more maxima whose locations are model-dependent. In the case of fibroblasts [18], interactions tend to favor parallel alignment which may be "head-to-head" $(\theta=0)$ or "head-to-tail" $(\theta=\pi)$. This can be modeled by taking a bimodal function of the form

$$
H(\theta)=\left[\cos 2 \theta-\cos 2 \theta_{0}\right]_{+}, \quad \theta_{0}<\frac{\pi}{4},
$$

where $[x]_{+}=x$ if $x>0$ and $[x]_{+}=0$ if $x \leq 0$, and $\theta_{0}$ determines the width of the two maxima. If only "head-to-head" alignment is favored, then $H(\theta)$ can be modeled by the unimodal function

$$
H(\theta)=\left[\cos \theta-\cos \theta_{0}\right]_{+}, \quad \theta_{0}<\frac{\pi}{2} .
$$

In the case of actin fibers [7], crosslinking proteins allow fibers to interact and bind at different configurations that include both parallel and orthogonal alignment. An example of an orthogonal interaction kernel is

$$
H(\theta)=\left[\cos 2 \theta_{0}-\cos 2 \theta\right]_{+}, \quad \theta_{0}>\frac{\pi}{4} .
$$

All three cases are illustrated in Figure 2.2. The final factor $\Delta$ is expected to be a positive function that is greater for coaligned elements than for flanking elements (at least in the case of parallel alignment). One possibility is to take

$$
\Delta(\psi)=1+\beta \cos 2 \psi, \quad \beta<1 .
$$



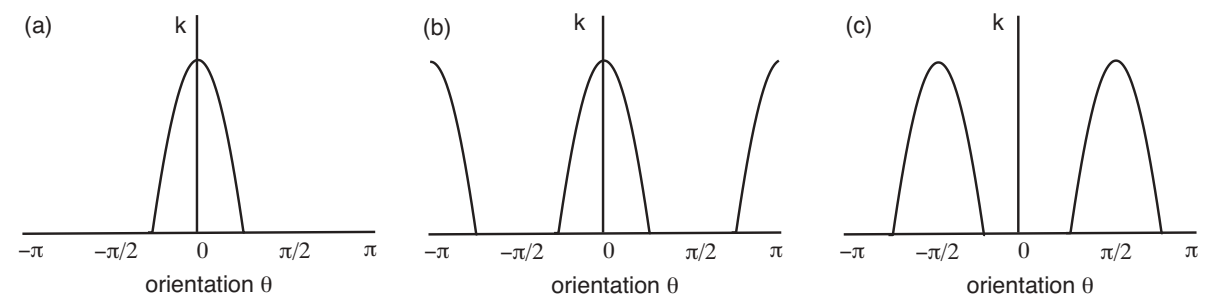

FIG. 2.2. Orientation kernel $H(\theta)$ for different favored alignments: (a) unimodal parallel alignment, (b) bimodal parallel alignment, (c) orthogonal alignment.

Note that as it stands the interaction kernel (2.8) is not symmetric under the interchange $(\mathbf{r}, \theta) \leftrightarrow\left(\mathbf{r}^{\prime}, \theta^{\prime}\right)$. It is straightforward to modify the kernel so that it has such an exchange symmetry by taking

$$
W\left(\mathbf{r}, \theta \mid \mathbf{r}^{\prime}, \theta^{\prime}\right)=\frac{1}{2} H\left(\theta-\theta^{\prime}\right)\left[J\left(\mathbf{r}-\mathbf{r}^{\prime}, \theta\right)+J\left(\mathbf{r}-\mathbf{r}^{\prime}, \theta^{\prime}\right)\right],
$$

where

$$
J(\mathbf{r}, \theta)=G(|\mathbf{r}|) \Delta(\arg (\mathbf{r})-\theta) .
$$

2.2. Euclidean symmetry. We now show that the nonseparable interaction kernel $W$ given by (2.8) is invariant under the action of the Euclidean group $\mathbf{E}(2)$, which is composed of the (semidirect) product of $\mathbf{O}(2)$, the group of planar rotations and reflections, with $\mathbf{R}^{2}$, the group of planar translations. The action of the Euclidean group on $\mathbf{R}^{2} \times \mathbf{S}^{1}$ is generated by

$$
\begin{aligned}
& \mathbf{s} \cdot(\mathbf{r}, \theta)=(\mathbf{r}+\mathbf{s}, \theta), \quad \mathbf{s} \in \mathbf{R}^{2}, \\
& \varphi \cdot(\mathbf{r}, \theta)=\left(R_{\varphi} \mathbf{r}, \theta+\varphi\right), \quad \varphi \in \mathbf{S}^{1}, \\
& \kappa \cdot(\mathbf{r}, \theta)=(\kappa \mathbf{r},-\theta),
\end{aligned}
$$

where $\kappa$ is the reflection $\left(x_{1}, x_{2}\right) \mapsto\left(x_{1},-x_{2}\right)$ and $R_{\varphi}$ is a rotation by $\varphi$. The corresponding group action on a function $a: \mathbf{R}^{2} \times \mathbf{S}^{1} \rightarrow \mathbf{R}$ is given by

$$
\gamma \cdot a(P)=a\left(\gamma^{-1} \cdot P\right) \quad \text { for all } \gamma \in \mathbf{O}(2) \dot{+} \mathbf{R}^{2},
$$

where $P=(\mathbf{r}, \theta)$, and the action on $W\left(P \mid P^{\prime}\right)$ is

$$
\gamma \cdot W\left(P \mid P^{\prime}\right)=W\left(\gamma^{-1} \cdot P \mid \gamma^{-1} \cdot P^{\prime}\right) .
$$

The so-called shift-twist action in (2.16) reflects a crucial feature of the underlying interactions, namely that they tend to favor collinear parallel elements. This correlation between relative angular position and object orientation means that invariance of $W$ under $\mathbf{E}(2)$ requires a rotation in the plane according to the twist $\mathbf{r} \rightarrow R_{\varphi} \mathbf{r}$ and a simultaneous rotation of object orientation according to the shift $\theta \rightarrow \theta+\varphi$ (see Figure 2.3). A similar argument holds for reflections.

Translation invariance of $W$ given by (2.8) follows immediately from the spatial homogeneity of the interactions, which implies that

$$
W\left(\mathbf{r}-\mathbf{s}, \theta \mid \mathbf{r}^{\prime}-\mathbf{s}, \theta^{\prime}\right)=W\left(\mathbf{r}, \theta \mid \mathbf{r}^{\prime}, \theta^{\prime}\right) .
$$



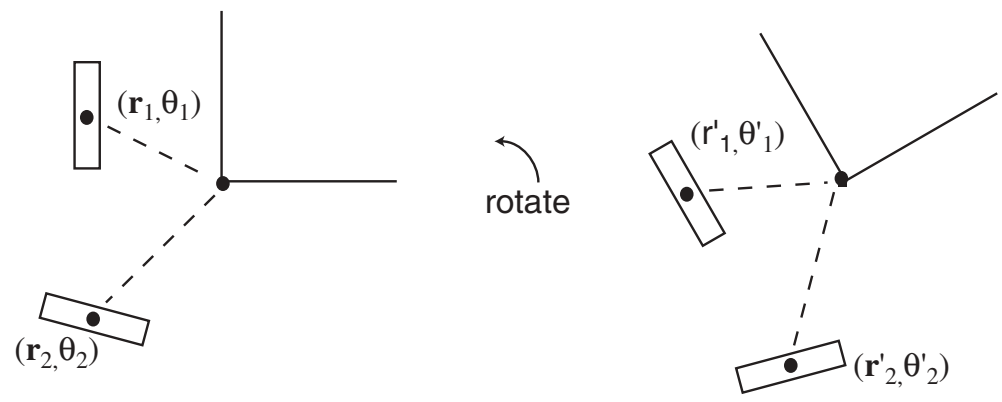

FIG. 2.3. Action of a rotation by $\varphi$ on two oriented objects located at planar positions $\mathbf{r}_{1}, \mathbf{r}_{2}$ and with internal orientations $\theta_{1}, \theta_{2}$. The action is of the form $(\mathbf{r}, \theta) \rightarrow\left(\mathbf{r}^{\prime}, \theta^{\prime}\right)=$ $\left(R_{\varphi} \mathbf{r}, \varphi+\theta\right)$.

Invariance with respect to a rotation by $\varphi$ follows from

$$
\begin{aligned}
W( & \left.R_{-\varphi} \mathbf{r}, \theta-\varphi \mid R_{-\varphi} \mathbf{r}^{\prime}, \theta^{\prime}-\varphi\right) \\
& =G\left(\left|R_{-\varphi}\left(\mathbf{r}-\mathbf{r}^{\prime}\right)\right|\right) H\left(\theta-\varphi-\theta^{\prime}+\varphi\right) \Delta\left(\arg \left[R_{-\varphi}\left(\mathbf{r}-\mathbf{r}^{\prime}\right)\right]-\theta+\varphi\right) \\
& =G\left(\left|\mathbf{r}-\mathbf{r}^{\prime}\right|\right) H\left(\theta-\theta^{\prime}\right) \Delta\left(\arg \left(\mathbf{r}-\mathbf{r}^{\prime}\right)-\theta\right) \\
& =W\left(\mathbf{r}, \theta \mid \mathbf{r}^{\prime}, \theta^{\prime}\right) .
\end{aligned}
$$

We have used the conditions $\left|R_{\varphi} \mathbf{r}\right|=|\mathbf{r}|$ and $\arg \left(R_{-\varphi} \mathbf{r}\right)=\arg (\mathbf{r})-\varphi$. Finally, invariance under a reflection $\kappa$ about the $x$-axis holds since

$$
\begin{aligned}
W\left(\kappa \mathbf{r},-\theta \mid \kappa \mathbf{r}^{\prime},-\theta^{\prime}\right) & =G\left(\left|\kappa\left(\mathbf{r}-\mathbf{r}^{\prime}\right)\right|\right) H\left(-\theta+\theta^{\prime}\right) \Delta\left(\arg \left[\kappa\left(\mathbf{r}-\mathbf{r}^{\prime}\right)\right]+\theta\right) \\
& =G\left(\left|\mathbf{r}-\mathbf{r}^{\prime}\right|\right) H\left(\theta-\theta^{\prime}\right) \Delta\left(-\arg \left(\mathbf{r}-\mathbf{r}^{\prime}\right)+\theta\right) \\
& =W\left(\mathbf{r}, \theta \mid \mathbf{r}^{\prime}, \theta^{\prime}\right) .
\end{aligned}
$$

We have used the conditions $\arg (\kappa \mathbf{r})=-\arg (\mathbf{r}), H(-\theta)=H(\theta)$, and $\Delta(-\psi)=\Delta(\psi)$. Finally, using identical arguments, it is straightforward to show that the modified kernel (2.14) is also invariant under the Euclidean group action (2.16).

Let us now determine how (2.7) transforms under the shift-twist action of the Euclidean group. Introducing the transformed coordinates $(\widetilde{\mathbf{r}}, \widetilde{\theta})=\gamma^{-1}(\mathbf{r}, \theta)$ and setting $\widetilde{f}(\mathbf{r}, \theta)=f(\widetilde{\mathbf{r}}, \widetilde{\theta})$, etc., we see that $(2.7)$ becomes

$$
\frac{\partial \widetilde{f}}{\partial t}=D_{1} \frac{\partial^{2} \widetilde{f}}{\partial \widetilde{\theta}^{2}}+D_{2} \widetilde{\nabla}^{2} \widetilde{f}-\eta_{1} \frac{\partial}{\partial \widetilde{\theta}}\left(\widetilde{f} \frac{\partial \widetilde{W * f}}{\partial \widetilde{\theta}}\right)-\eta_{2} \widetilde{\nabla} \cdot(\widetilde{f} \widetilde{\nabla} \widetilde{W * f}) .
$$

Invariance of the weight kernel $W$ implies that $\widetilde{W * f}=W * \widetilde{f}$ :

$$
\begin{aligned}
W * f\left(\gamma^{-1} P, t\right) & =\int_{\mathbf{R}^{2} \times \mathbf{S}^{1}} W\left(\gamma^{-1} P \mid P^{\prime}\right) f\left(P^{\prime}, t\right) d P^{\prime} \\
& =\int_{\mathbf{R}^{2} \times \mathbf{S}^{1}} W\left(P \mid \gamma P^{\prime}\right) f\left(P^{\prime}, t\right) d P^{\prime} \\
& =\int_{\mathbf{R}^{2} \times \mathbf{S}^{1}} W\left(P \mid P^{\prime \prime}\right) f\left(\gamma^{-1} P^{\prime \prime}, t\right) d P^{\prime \prime},
\end{aligned}
$$

since $d\left[\gamma^{-1} P\right]= \pm d P$ and $W$ is Euclidean invariant. It is also easy to establish that 
all of the quadratic differential operators are Euclidean invariant, that is,

$$
\frac{\partial}{\partial \widetilde{\theta}}\left(a \frac{\partial}{\partial \widetilde{\theta}}\right)=\frac{\partial}{\partial \theta}\left(a \frac{\partial}{\partial \theta}\right), \quad \widetilde{\nabla} \cdot(a \widetilde{\nabla})=\nabla \cdot(a \nabla)
$$

for any scalar function $a(\mathbf{r}, \theta)$. Hence,

$$
\frac{\partial \widetilde{f}}{\partial t}=D_{1} \frac{\partial^{2} \widetilde{f}}{\partial \theta^{2}}+D_{2} \nabla^{2} \widetilde{f}-\eta_{1} \frac{\partial}{\partial \theta}\left(\widetilde{f} \frac{\partial W * \widetilde{f}}{\partial \widetilde{\theta}}\right)-\eta_{2} \nabla \cdot(\widetilde{f} \nabla W * \widetilde{f}) .
$$

If we rewrite (2.7) as an operator equation, namely,

$$
\mathcal{F}_{t}[f] \equiv \frac{d f}{d t}-\mathcal{F}[f]=0,
$$

then it follows that $\gamma \mathcal{F}_{t}[f]=\mathcal{F}_{t}[\gamma f]=\mathcal{F}_{t}[\widetilde{f}]$. Thus $\mathcal{F}_{t}$ commutes with $\gamma \in \mathbf{E}(2)$, and $\mathcal{F}_{t}$ is said to be equivariant with respect to the symmetry group $\mathbf{E}(2)$ (see [13]). In sections 3 and 4 we show how the equivariance of the operator $\mathcal{F}_{t}$ with respect to the shift-twist action of $\mathbf{E}(2)$ has major implications for the nature of solutions bifurcating from a homogeneous steady state solution. In particular, equivariance implies that there exist two distinct forms of spatio-angular order, which are associated with scalar and pseudoscalar representations of the Euclidean group. Further details concerning the general approach used in this paper, as well as many illustrative examples, can be found in the recent excellent book on the role of symmetry in nonlinear dynamical systems by Golubitsky and Stewart [13].

3. Linear stability analysis. The first step in the analysis of pattern forming instabilities is to linearize (2.7) about the homogeneous solution $f(\mathbf{r}, \theta)=\bar{f}$, where

$$
\bar{f}=\frac{N}{2 \pi A[\mathcal{D}]}, \quad A[\mathcal{D}]=\int_{\mathcal{D}} d^{2} \mathbf{r},
$$

and to solve the resulting eigenvalue problem. In particular, we wish to find conditions under which the homogeneous solution becomes marginally stable due to the vanishing of one of the (degenerate) eigenvalues, and to identify the marginally stable modes. In the following we will consider the modified version of the interaction kernel given by (2.14).

3.1. Eigenvalue equation. Substitute

$$
f(\mathbf{r}, \theta, t)=\bar{f}+a(\mathbf{r}, \theta) \mathrm{e}^{\lambda t}
$$

into (2.7) and expand to first order in $a$. This generates the linear eigenvalue equation

$$
\begin{aligned}
\lambda a & =\widehat{L} a \\
& \equiv D_{1} \frac{\partial^{2} a}{\partial \theta^{2}}+D_{2} \nabla^{2} a-\eta_{1} \bar{f} \frac{\partial^{2}(W * a)}{\partial \theta^{2}}-\eta_{2} \bar{f} \nabla^{2}(W * a) .
\end{aligned}
$$

Since the homogeneous solution has full Euclidean symmetry, $\gamma \bar{f}=\bar{f}$ for all $\gamma \in \mathbf{E}(2)$, it follows that the linear operator $\widehat{L}$ is equivariant with respect to the Euclidean group action (2.16). This can be shown either by explicitly using (3.3) or by rewriting (2.7) in the form (2.21) and exploiting the equivariance of $\mathcal{F}$. In the latter case, linearizing both sides of the equation $\gamma \mathcal{F}[f]=\mathcal{F}[\gamma f]$ about $\bar{f}$ gives

$$
\gamma(\mathcal{F}[\bar{f}]+D \mathcal{F}[\bar{f}](f-\bar{f}))=\mathcal{F}[\gamma \bar{f}]+D \mathcal{F}[\gamma \bar{f}](\gamma f-\bar{f}),
$$


which implies that $\gamma \widehat{L}=\widehat{L} \gamma$ for all $\gamma \in \mathbf{E}(2)$, where $\widehat{L}=D \mathcal{F}[\bar{f}]$. Equivariance of $\widehat{L}$ determines the basic form of the eigenfunction solutions of (3.3); namely, they are given by irreducible representations of the group action (2.16) on the space $\mathbf{R}^{2} \times \mathbf{S}^{1}$. We show this following similar arguments to those of Bressloff et al. [3, 4]. First, translation symmetry implies that the eigenfunctions can be expressed in the form

$$
a(\mathbf{r}, \theta)=u(\theta-\varphi) e^{i \mathbf{k} \cdot \mathbf{r}}+\text { c.c. },
$$

where c.c. denotes the complex conjugate, $\mathbf{k}=q(\cos \varphi, \sin \varphi)$, and

$$
\begin{aligned}
\lambda u(\theta) & =D_{1} \frac{\partial^{2} u(\theta)}{\partial \theta^{2}}-D_{2} q^{2} u(\theta) \\
& -\frac{\bar{f}}{2}\left[\eta_{1} \frac{\partial^{2}}{\partial \theta^{2}}-\eta_{2} q^{2}\right] \int_{-\pi}^{\pi} H\left(\theta-\theta^{\prime}\right)\left[\widehat{J}(\mathbf{k}, \theta+\varphi)+\widehat{J}\left(\mathbf{k}, \theta^{\prime}+\varphi\right)\right] u\left(\theta^{\prime}\right) \frac{d \theta^{\prime}}{2 \pi} .
\end{aligned}
$$

Here $\widehat{J}(\mathbf{k}, \theta)$ is the Fourier transform of $J(\mathbf{r}, \theta)$,

$$
\widehat{J}(\mathbf{k}, \theta)=\int_{\mathbf{R}^{2}} \mathrm{e}^{-i \mathbf{k} \cdot \mathbf{r}} J(\mathbf{r}, \theta) d^{2} \mathbf{r} .
$$

Euclidean symmetry further restricts the structure of the eigensolutions $u(\theta)$ of (3.5) as follows.

(i) The Fourier transform $\widehat{J}(\mathbf{k}, \theta+\varphi)$ is independent of the direction $\varphi=\arg (\mathbf{k})$. This is easy to establish as follows:

$$
\begin{aligned}
\widehat{J}(\mathbf{k}, \theta+\varphi) & =\int_{\mathbf{R}^{2}} \mathrm{e}^{-i \mathbf{k} \cdot \mathbf{r}} J(\mathbf{r}, \theta+\varphi) d^{2} \mathbf{r} \\
& =\int_{0}^{\infty} \int_{-\pi}^{\pi} \mathrm{e}^{-i q r \cos (\psi-\varphi)} G(r) \Delta(\psi-\theta-\varphi) d \psi r d r \\
& =\int_{0}^{\infty} \int_{-\pi}^{\pi} \mathrm{e}^{-i q r \cos (\psi)} G(r) \Delta(\psi-\theta) d \psi r d r \\
& =\widehat{J}(q, \theta) .
\end{aligned}
$$

Therefore, $\lambda$ and $u(\theta)$ depend only on the magnitude $q=|\mathbf{k}|$ of the wavevector $\mathbf{k}$, and there is an infinite degeneracy due to rotational invariance. Note, however, that the eigenfunction (3.4) depends on $u(\theta-\varphi)$, which reflects the shift-twist action of the rotation group.

(ii) For each $\mathbf{k}$ the associated subspace of eigenfunctions

$$
V_{\mathbf{k}}=\left\{u(\theta-\varphi) \mathrm{e}^{i \mathbf{k} \cdot \mathbf{r}}+\text { c.c. }\right\}
$$

decomposes into two invariant subspaces,

$$
V_{\mathbf{k}}=V_{\mathbf{k}}^{+} \oplus V_{\mathbf{k}}^{-},
$$

corresponding to even and odd functions, respectively:

$$
V_{\mathbf{k}}^{+}=\left\{v \in V_{\mathbf{k}}: u(-\theta)=u(\theta)\right\} \quad \text { and } \quad V_{\mathbf{k}}^{-}=\left\{v \in V_{\mathbf{k}}: u(-\theta)=-u(\theta)\right\} .
$$

This is a consequence of reflection invariance, as we now indicate. That is, let $\kappa_{\mathbf{k}}$ denote reflections about the wavevector $\mathbf{k}$ so that $\kappa_{\mathbf{k}} \mathbf{k}=\mathbf{k}$. Then $\kappa_{\mathbf{k}} a(\mathbf{r}, \phi)=$ 
$a\left(\kappa_{\mathbf{k}} \mathbf{r}, 2 \varphi-\phi\right)=u(\varphi-\phi) \mathrm{e}^{i \mathbf{k} \cdot \mathbf{r}}+$ c.c. Since $\kappa_{\mathbf{k}}$ is a reflection, any space that it acts on decomposes into two subspaces - one on which it acts as the identity $I$ and one on which it acts as $-I$. The even and odd functions correspond to scalar and pseudoscalar representations of the Euclidean group studied in a more general context by Vivancos, Chossat, and Melbourne [1].

A further reduction of (3.5) can be achieved by expanding the $2 \pi$-periodic function $u(\theta)$ as a Fourier series with respect to $\theta$ :

$$
u(\theta)=\sum_{n \in \mathbf{Z}} U_{n} \mathrm{e}^{i n \theta} .
$$

This then leads to the matrix eigenvalue equation

$$
\lambda(q) U_{n}=[\mathbf{L}(q) \mathbf{U}]_{n} \equiv-A_{n}(q) U_{n}+\frac{1}{2} B_{n}(q) \sum_{m \in \mathbf{Z}} \widehat{J}_{n-m}(q)\left[H_{m}+H_{n}\right] U_{m},
$$

where

$$
A_{n}(q)=D_{1} n^{2}+D_{2} q^{2}, \quad B_{n}(q)=\bar{f}\left(\eta_{1} n^{2}+\eta_{2} q^{2}\right)
$$

and

$$
\widehat{J}_{n}(q)=\int_{-\pi}^{\pi} \mathrm{e}^{-i n \theta} \widehat{J}(q, \theta) \frac{d \theta}{2 \pi}=\Delta_{n} \widehat{G}_{n}(q)
$$

with

$$
\widehat{G}_{n}(q)=\int_{0}^{\infty} \int_{-\pi}^{\pi} \mathrm{e}^{-i q r \cos (\psi)} \mathrm{e}^{-i n \psi} G(r) d \psi r d r .
$$

We have used (3.7) together with the Fourier series expansions

$$
H(\theta)=\sum_{n \in \mathbf{Z}} \mathrm{e}^{i n \theta} H_{n}, \quad \Delta(\psi)=\sum_{n \in \mathbf{Z}} \mathrm{e}^{i n \psi} \Delta_{n} .
$$

Note that $H_{n}^{*}=H_{-n}=H_{n}$ and $\Delta_{n}^{*}=\Delta_{-n}=\Delta_{n}$, since $H(\theta)$ and $\Delta(\theta)$ are assumed to be real even functions of $\theta$. Equations (3.14) and (3.15) imply that

$$
\widehat{J}_{n}(q)^{*}=(-1)^{n} \widehat{J}_{-n}(q), \quad \widehat{J}_{-n}(q)=\widehat{J}_{n}(q) .
$$

Denote the set of solutions to (3.12) by $\left\{\left(\lambda_{j}(q), \mathbf{U}_{j}(q)\right), j \in \mathbf{Z}\right\}$. We now establish conditions under which the eigenvalues $\lambda_{j}(q)$ are real for all $q \in \mathbf{R}$. The case $q=0$ is trivial because $\widehat{J}_{n}(0) \sim \delta_{n, 0}$ so that

$$
\lambda_{n}(0)=-A_{n}(0)+B_{n}(0) H_{n} .
$$

Therefore, we take $q \neq 0$. Introduce the inner product of two periodic functions $V(\theta), U(\theta)$ according to

$$
\langle V \mid U\rangle=\int_{-\pi}^{\pi} V^{*}(\theta) U(\theta) \frac{d \theta}{2 \pi}=\sum_{n \in \mathbf{Z}} V_{n}^{*} U_{n}=\langle\mathbf{V} \mid \mathbf{U}\rangle,
$$


where $V^{*}$ denotes the complex conjugate of $V$. The adjoint matrix $\mathbf{L}(q)^{\dagger}$ is then given by

$$
\left[\mathbf{L}(q)^{\dagger} \mathbf{V}\right]_{n} \equiv-A_{n}(q) V_{n}+\frac{1}{2} \sum_{m \in \mathbf{Z}} \widehat{J}_{m-n}(q)^{*} B_{m}(q)\left[H_{m}+H_{n}\right] V_{m}
$$

Equation (3.17) implies that $\mathbf{L}(q)^{\dagger}$ and $\mathbf{L}(q)$ have the same set of eigenvalues,

$$
\mathbf{L}(q) \mathbf{U}_{j}(q)=\lambda_{j}(q) \mathbf{U}_{j}(q), \quad \mathbf{L}(q)^{\dagger} \mathbf{V}_{j}(q)=\lambda_{j}(q) \mathbf{V}_{j}(q),
$$

with the corresponding eigenvectors related according to

$$
U_{j, n}(q)=(-1)^{n} B_{n}(q) V_{j, n}(q) .
$$

This relationship is invertible since $B_{n}(q)>0$ for all $n \in \mathbf{Z}$ and $q \neq 0$. It further follows that

$$
\begin{aligned}
\lambda_{j}\left\langle\mathbf{V}_{j^{\prime}} \mid \mathbf{U}_{j}\right\rangle & =\left\langle\mathbf{V}_{j^{\prime}} \mid \mathbf{L} \mathbf{U}_{j}\right\rangle \\
& =\left\langle\mathbf{L}^{\dagger} \mathbf{V} \mid \mathbf{U}\right\rangle \\
& =\lambda_{j^{\prime}}^{*}\left\langle\mathbf{V}_{j^{\prime}} \mid \mathbf{U}_{j}\right\rangle
\end{aligned}
$$

Hence, if $\lambda_{j}(q) \neq \lambda_{j^{\prime}}(q)$ for $j \neq j^{\prime}$, then the vectors $\mathbf{U}_{j}(q)$ and $\mathbf{V}_{j}(q)$ form a biorthogonal system with

$$
\left\langle\mathbf{V}_{j^{\prime}}(q) \mid \mathbf{U}_{j}(q)\right\rangle=\chi_{j}(q) \delta_{j, j^{\prime}}
$$

and

$$
\chi_{j}(q)=\left\langle\mathbf{V}_{j}(q) \mid \mathbf{U}_{j}(q)\right\rangle=\sum_{n}(-1)^{n} B_{n}(q)\left|V_{j, n}(q)\right|^{2} .
$$

It also follows that $\lambda_{j}(q)=\lambda_{j}^{*}(q)$ if $\chi_{j}(q) \neq 0$. The latter condition certainly holds for all $q \neq 0$ and $j \in \mathbf{Z}$ when $\Delta(\psi)$ is $\pi$-periodic,

$$
\Delta(\psi)=\Delta(\psi+\pi)
$$

corresponding to the situation in which collinear interactions are equally strong at the front and at the back (see Figure 2.1(b)). In this case $\Delta_{n}=0$ and $\widehat{J}_{n}(q)=0$ for all odd integers $n$, and $\mathbf{L}(q)$ becomes a real matrix that only couples together even-to-even or odd-to-odd components $U_{n}$. Hence, $\chi_{j}(q)= \pm \sum_{n}^{ \pm} B_{n}(q)\left|V_{j, n}(q)\right|^{2} \neq 0$, where $\sum_{n}^{ \pm}$denotes the sum over even and odd integers, respectively. The corresponding eigenfunctions satisfy either $U(\theta+\pi)=U(\theta)$ or $U(\theta+\pi)=-U(\theta)$ and can be taken to be real-valued. If $\Delta(\psi)$ is not $\pi$-periodic, then the eigenvalues $\lambda_{j}(q)$ are still real, provided that $\chi_{j}(q) \neq 0$ except at isolated points, which follows from the observation that $\lambda_{j}(q)$ is a continuous function of $q$. In this more general situation, however, the eigenfunctions $U(\theta)$ will be complex-valued.

3.2. Perturbation expansion. The calculation of the eigenvalues and eigenfunctions of the linearized equation (3.5), and hence the derivation of conditions for the marginal stability of the homogeneous state, has been reduced to the problem of solving the matrix equation (3.12). In general it is not possible to solve this equation exactly. Here we will carry out a perturbation expansion under the assumption that 
the dependence of the interactions on relative direction in the plane is weak. In other words, we write

$$
\Delta(\psi)=1+\beta \Psi(\psi), \quad 0 \leq \beta \ll 1, \quad|\Psi(\psi)| \leq 1 \text { for all } \psi,
$$

with $\Psi_{0}=\int_{-\pi}^{\pi} \Psi(\psi) d \psi=0$. Equation (3.12) can then be rewritten in the form

$$
\left[\lambda(q)-W_{n}(q)\right] U_{n}=\beta \sum_{n \in \mathbf{Z}} \widehat{W}_{n m}(q) U_{m}
$$

where

$$
W_{n}(q)=-A_{n}(q)+B_{n}(q) H_{n} \widehat{G}_{0}(q)
$$

and

$$
\widehat{W}_{n m}(q)=\frac{1}{2} B_{n}(q)\left[H_{m}+H_{n}\right] \Psi_{n-m} \widehat{G}_{n-m}(q)
$$

with $A_{n}(q), B_{n}(q), \widehat{G}_{n}(q)$ given by (3.13) and (3.15). Equation (3.28) can then be solved by expanding as a power series in $\beta$ and using degenerate perturbation theory.

Case $\beta=0$. In the limiting case that the interaction kernel is independent of relative direction in the plane, $\beta=0,(3.28)$ reduces to the result previously obtained for separable kernels [18]: the eigenvalues are

$$
\lambda_{n}(q)=W_{n}(q)=-\left(D_{1} n^{2}+D_{2} q^{2}\right)+\bar{f}\left(\eta_{1} n^{2}+\eta_{2} q^{2}\right) H_{n} \mathrm{e}^{-q^{2} / 2}
$$

with corresponding eigenfunctions

$$
a_{n, \mathbf{k}}(\mathbf{r}, \theta)=\mathrm{e}^{i n \theta} \mathrm{e}^{i \mathbf{k} \cdot \mathbf{r}}, \quad|\mathbf{k}|=q .
$$

We have used the result $\widehat{G}_{0}(q)=\mathrm{e}^{-q^{2} / 2}$, which follows from substituting (2.9) into (3.15) and evaluating the resulting Gaussian integral. Note that the full interaction kernel $W$ is invariant with respect to the group $\mathbf{E}(2) \times \mathbf{O}(2)$ so that the odd and even modes $a_{n, \mathbf{k}} \pm a_{-n, \mathbf{k}}$ are degenerate, that is, $\lambda_{-n}(q)=\lambda_{n}(q)$.

Case $\beta>0$. For nonzero $\beta$, there is a $q$-dependent splitting of the pair of degenerate eigenvalues $\lambda_{ \pm n}(q), n \neq 0$, which separates out odd and even solutions. Denoting the characteristic size of such a splitting by $\delta \lambda=\mathcal{O}(\beta)$, we impose the condition that $\delta \lambda \ll \Delta W$, where $\Delta W=\min \left\{W_{n}-W_{m}, m \neq \pm n\right\}$. This ensures that the perturbation does not excite states associated with other eigenvalues of the unperturbed problem. We can then restrict ourselves to calculating perturbative corrections to the degenerate eigenvalues $\lambda_{ \pm n}$ and their associated eigenfunctions. Therefore, introduce the power series expansions

$$
\lambda_{ \pm n}=W_{n}+\beta \lambda_{ \pm n}^{(1)}+\beta^{2} \lambda_{ \pm n}^{(2)}+\cdots
$$

and

$$
U_{ \pm n, m}=z_{ \pm n} \delta_{m, \pm n}+\beta U_{ \pm n, m}^{(1)}+\beta^{2} U_{ \pm n, m}^{(2)}+\cdots,
$$

where $\delta_{n, m}$ is the Kronecker delta function. Here $U_{n, m}$ is the $m$ th component of the vector $\mathbf{U}_{n}$ associated with the eigenvalue $\lambda_{n}$. Substitute these expansions into the matrix eigenvalue equation (3.28) and systematically solve the resulting hierarchy of 
equations to successive orders in $\beta$ using degenerate perturbation theory along similar lines to [3]. This leads to the following results:

(i) the even $(+)$ and odd $(-)$ eigenvalues to $\mathcal{O}\left(\beta^{2}\right)$ are

$$
\begin{aligned}
& \lambda_{ \pm n}(q)=W_{n}(q)+\beta\left[\widehat{W}_{n n}(q) \pm \widehat{W}_{n,-n}(q)\right] \\
&+\beta^{2} \sum_{0 \leq m \neq n} \frac{\left[\widehat{W}_{n m}(q) \pm \widehat{W}_{-n, m}(q)\right]\left[\widehat{W}_{m n}(q) \pm \widehat{W}_{m,-n}(q)\right]}{W_{n}-W_{m}}
\end{aligned}
$$

(ii) the corresponding eigenfunctions to $\mathcal{O}(\beta)$ are

$$
\begin{aligned}
& a_{n, \mathbf{k}}(\mathbf{r}, \theta)=\left[\cos (n \theta)+\beta \sum_{0 \leq m \neq n} U_{m}^{+}(q) \cos (m \theta)\right] \mathrm{e}^{i \mathbf{k} \cdot \mathbf{r}} \\
& a_{-n, \mathbf{k}}(\mathbf{r}, \theta)=\left[\sin (n \theta)+\beta \sum_{0<m \neq n} U_{m}^{-}(q) \sin (m \theta)\right] \mathrm{e}^{i \mathbf{k} \cdot \mathbf{r}}
\end{aligned}
$$

with $|\mathbf{k}|=q$ and

$$
U_{0}^{+}(q)=\frac{\widehat{W}_{0 n}(q)}{W_{n}-W_{0}}, \quad U_{m}^{ \pm}(q)=\frac{\widehat{W}_{m n}(q) \pm \widehat{W}_{m,-n}(q)}{W_{n}-W_{m}}, \quad 0<m \neq n .
$$

It is important to stress that the splitting of even and odd branches for $\beta>0$ is a consequence of the underlying shift-twist symmetry and thus occurs beyond the small $\beta$ regime.

3.3. Marginal stability. We now determine the marginal stability boundaries in parameter space that separate regions of stability from regions of instability. Crossing one of these boundaries signals that one or more eigenvalues become positive and their corresponding eigenfunctions start to grow, leading to the formation of a selforganizing pattern. (Conservation of population number implies that there always exists one neutrally stable mode $q=0, n=0$, that is, $\lambda_{0}(0)=0$.) For concreteness, we treat the rescaled diffusion coefficients $D_{1} \rightarrow D_{1} \bar{f}, D_{2} \rightarrow D_{2} \bar{f}$ as bifurcation parameters. An adiabatic increase in the mean density $\bar{f}$ due to a growth in population number or a contraction in the area occupied by the population then corresponds to a reduction in $D_{1}$ and $D_{2}$. This reduction can lead to one of four distinct types of instability, depending on which eigenmodes are first excited:

(I) if $q \neq 0, n=0$, then a spatially periodic pattern forms without any angular order (aggregation without orientation);

(II) if $q=0, n \neq 0$, then a pattern with angular order forms that is spatially uniform (orientation without aggregation);

(IIIa,b) if $q \neq 0, n \neq 0$, then a pattern with spatio-angular order forms, in which the angle of preferred orientation changes periodically in space even though the spatial density remains homogeneous. The invariance of the interaction kernel under Euclidean shift-twist symmetry implies that when $\beta>0$, there are two kinds of spatio-angular patterns, corresponding to (a) even and (b) odd eigenmodes, respectively. Examples of such eigenmodes are shown in Figure 3.1 under the simplifying assumption that a particular harmonic component $n$ dominates. The crucial observation is that the direction of preferred orientation is correlated with the direction 


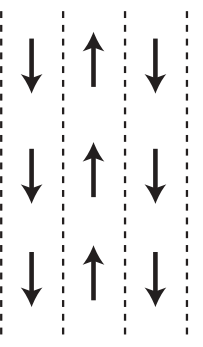

$\mathrm{n}=1$ (odd)

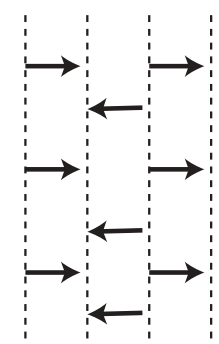

$\mathrm{n}=1$ (even)

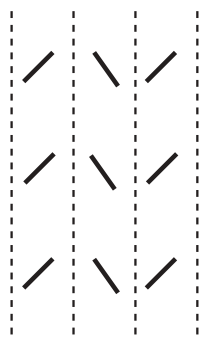

$\mathrm{n}=2$ (odd)

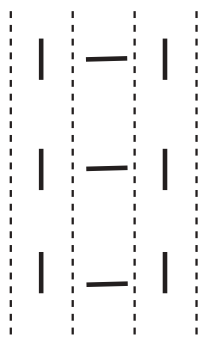

$\mathrm{n}=2$ (even)

FIG. 3.1. Examples of even $\left(a_{n, \mathbf{k}}=\cos (n \theta) \mathrm{e}^{i \mathbf{k} \cdot \mathbf{r}}+\right.$ c.c. $)$ and odd $\left(a_{n, \mathbf{k}}=\sin (n \theta) \mathrm{e}^{i \mathbf{k} \cdot \mathbf{r}}+\right.$ c.c. $)$ eigenmodes with spatio-angular order in the case of a horizontal wavevector $\mathbf{k}=(q, 0)$. If $n=1$, then there is a unique direction of preferred orientation (indicated by an arrow). If $n=2$, then there are two preferred directions of motion (indicated by a bar). The width of each vertical strip is $\pi / q$.

of the wavevector $\mathbf{k}$, whereas there is no such correlation when $\beta=0$ so that the distinction between odd and even solutions disappears.

3.3.1. Separable interaction kernel $(\boldsymbol{\beta}=0)$. Before determining the effects of shift-twist symmetry on pattern forming instabilities of the homogeneous state, we first discuss the separable case previously analyzed by Mogilner and Edelstein-Keshet [18]. The eigenvalues for $\beta=0$ are given by (3.31). In particular, when $n=0$ we have (on setting $\bar{f}=1$ )

$$
\lambda_{0}(q)=q^{2}\left(-D_{2}+\eta_{2} H_{0} \mathrm{e}^{-q^{2} / 2}\right) .
$$

It is clear that $\lambda_{0}(q)<0$ for all $q \neq 0$ if $D_{2}>D_{2,0}$, where

$$
D_{2,0}=\eta_{2} H_{0} .
$$

If $D_{2}<D_{2,0}$, then the homogeneous state is unstable with respect to the excitation of eigenmodes over a range of wavenumbers that includes the origin so that $q \approx$ $0, n=0$. Although this is a type I instability, the emerging pattern tends to involve long-wavelength spatial inhomogeneities. Therefore, the resulting stationary state could be treated as homogeneous on shorter spatial scales and thus be susceptible to secondary bifurcations associated with excitation of modes with $q \neq 0, n \neq 0$ (see below). Alternatively, the $n=0$ modes could be stabilized by incorporating an additional contribution to the interaction kernel $W$ in the form of a repulsive hard-core potential:

$$
W\left(\mathbf{r}, \theta \mid \mathbf{r}^{\prime}, \theta^{\prime}\right)=G\left(\mathbf{r}-\mathbf{r}^{\prime}\right) H\left(\theta-\theta^{\prime}\right) \Delta\left(\arg \left(\mathbf{r}-\mathbf{r}^{\prime}\right)-\theta\right)-C \delta\left(\mathbf{r}-\mathbf{r}^{\prime}\right)
$$

with $C>0$. This modifies the $\beta=0$ contribution to $\lambda_{0}$ according to

$$
\lambda_{0}(q)=q^{2}\left(-D_{2}+\eta_{2} H_{0} \mathrm{e}^{-q^{2} / 2}-H_{0} C\right),
$$

and the stability condition becomes $D_{2}>D_{2,0}^{\prime}$ with

$$
D_{2,0}^{\prime}=H_{0}\left(\eta_{2}-C\right) \text {. }
$$


Now consider the branch of eigenmodes for a given $n, n \neq 0$, such that $H_{n}>0$ (otherwise $\lambda_{n}(q)<0$ for all $q$ ). Differentiating (3.31) with respect to $q$ gives

$$
\frac{d \lambda_{n}(q)}{d q}=q\left[-2 D_{2}+H_{n}\left(2 \eta_{2}-\left(\eta_{1} n^{2}+\eta_{2} q^{2}\right)\right) \mathrm{e}^{-q^{2} / 2}\right] .
$$

This implies that $\lambda_{n}(q)$ has a maximum at $q=0$ when $D_{2}>D_{2, n}$ and a maximum at $q=q_{c} \neq 0$ when $D_{2}<D_{2, n}$, where

$$
D_{2, n}=H_{n}\left(\eta_{2}-\eta_{1} n^{2} / 2\right) .
$$

Since

$$
\lambda_{n}(0)=n^{2}\left[-D_{1}+\eta_{1} H_{n}\right],
$$

it follows that $\lambda_{n}(q)<0$ for all $n \neq 0$ when $D_{2}>D_{2, n}$ and $D_{1}>D_{1, n}$ with

$$
D_{1, n}=\eta_{1} H_{n} .
$$

On the other hand, if $D_{2}>D_{2, n}$ and $D_{1}<D_{1, n}$, then there is a type II instability due to excitation of the eigenmode at $q=0$. Finally, if $0<D_{2}<D_{2, n}$, then there is a type III instability due to excitation of the eigenmodes with wavenumber $q=q_{c} \neq 0$. This occurs at a critical value $D_{1}=\mathcal{F}_{n}\left(D_{2}\right)$ with $\mathcal{F}_{n}$ a monotonically decreasing function such that $\mathcal{F}_{n}\left(D_{2, n}\right)=D_{1, n}$ and $\mathcal{F}_{n}(0)=D_{1, n}^{\prime}>D_{1, n}$. Note that this last instability can occur only for integers $n$ satisfying $n^{2}<2 \eta_{2} / \eta_{1}$.

In order to determine the stability of the homogeneous state one now has to combine the stability conditions for all $n$. For concreteness, suppose the set of coefficients $\left\{H_{n}, n \neq 0\right\}$ has a maximum at $n=n_{c}$ such that $D_{j, n_{c}}>D_{j, n}$ for all $n \neq 0, n_{c}$. This leads to the stability diagram shown in Figure 3.2. In the absence of a repulsive contribution to the interaction kernel, the region of stability is given by the dark shaded region in Figure 3.2. Crossing the vertical boundary at $D_{1}=D_{1, n_{c}}$ induces a type II instability, whereas crossing the horizontal boundary at $D_{2}=D_{2,0}$ induces a type I instability. A type III instability can occur only through a secondary bifurcation. On the other hand, when there is a repulsive contribution to the potential, the stability region extends to include both the dark and light shaded regions. A type II instability now occurs on crossing the lower horizontal boundary at $D_{2}=D_{2,0}^{\prime}$, so that there is an additional curved boundary $D_{1}=\mathcal{F}_{n_{c}}\left(D_{2}\right)$ for $D_{2,0}^{\prime}<D_{2}<D_{2, n_{c}}$. Crossing this boundary induces a type III instability, but there is no separation into even or odd patterns, since the separable interaction kernel is invariant under the standard Euclidean group action.

3.3.2. Nonseparable interaction kernel $(\boldsymbol{\beta}>0)$. We now show that patterns with even or odd spatio-angular order can occur when $\beta>0$. We take $D_{1}$ and $D_{2}$ to be close to the curved boundary of the stability region shown in Figure 3.2 , where the unperturbed system undergoes a type III instability, and consider $\mathcal{O}(\beta)$ corrections to the eigenvalues $\lambda_{ \pm n}(q)$ for $n=n_{c}$. Using (3.30) and (3.35) with $\Psi_{0}=0$, we find that $\lambda_{ \pm n}(q)=-D_{1} n^{2}+\Lambda_{ \pm n}(q)+\mathcal{O}\left(\beta^{2}\right)$, where

$$
\Lambda_{ \pm n}(q)=-D_{2} q^{2}+\left(\eta_{1} n^{2}+\eta_{2} q^{2}\right) H_{n}\left[\mathrm{e}^{-q^{2} / 2} \pm \beta \Psi_{2 n} \widehat{G}_{2 n}(q)\right]
$$

Suppose that $\Lambda_{ \pm n}(q)$ has a unique maximum at $q=q_{ \pm} \neq 0$. If $\Lambda_{n}\left(q_{+}\right)>\Lambda_{-n}\left(q_{-}\right)$, then the homogeneous state will become unstable at the critical point $D_{1}=\Lambda_{n}\left(q_{+}\right) / n^{2}$ 


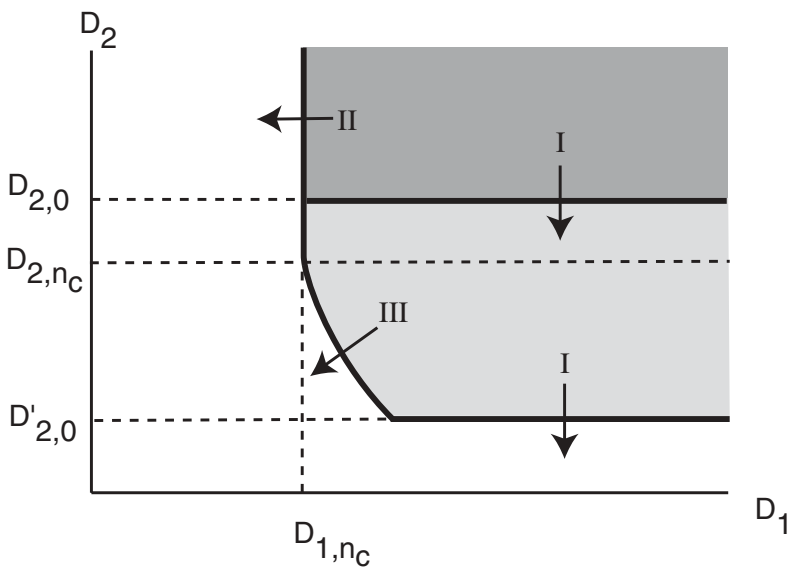

FIG. 3.2. Stability diagram for the homogeneous state when $\beta=0$. In the absence of a repulsive contribution to the interaction kernel, the region of stability is given by the dark shaded region. This extends to include the light shaded region when repulsion is included. The type of primary instability induced by crossing each boundary of the stability region is also indicated. (See text for details.)

due to excitation of even eigenmodes with wavenumber $q_{+}$, whereas if $\Lambda_{-n}\left(q_{-}\right)>$ $\Lambda_{n}\left(q_{+}\right)$, then the homogeneous state will become unstable at the critical point $D_{1}=$ $\Lambda_{-n}\left(q_{-}\right) / n^{2}$ due to excitation of odd eigenmodes with wavenumber $q_{-}$.

In order to determine whether an odd or even pattern arises, it is necessary to evaluate the function $\widehat{G}_{2 n}(q)$ for the given Gaussian kernel (2.9). Rewriting (3.15) for even integers as

$$
\widehat{G}_{2 n}(q)=\int_{0}^{\pi} \mathrm{e}^{-2 i n \psi}\left[\int_{0}^{\infty} G(r) \cos (r q \cos \psi) r d r\right] d \psi
$$

and using the Jacobi-Anger expansion

$$
\cos (s q \cos \psi)=J_{0}(s q)+2 \sum_{m=1}^{\infty}(-1)^{m} J_{2 m}(s q) \cos (2 m \psi),
$$

with $J_{n}(x)$ the Bessel function of integer order $n$, we find that $\widehat{G}_{2 n}$ is related to $G$ according to

$$
\widehat{G}_{2 n}(q)=(-1)^{n} \int_{0}^{\infty} G(r) J_{2 n}(r q) r d r .
$$

Substituting (2.9) into (3.49) and using standard properties of Bessel functions leads to the result

$$
\widehat{G}_{2 n}(q)=\frac{q \sigma \sqrt{2 \pi}}{4} \mathrm{e}^{-\sigma^{2} q^{2} / 4}\left[I_{n-1 / 2}\left(\frac{\sigma^{2} q^{2}}{4}\right)-I_{n+1 / 2}\left(\frac{\sigma^{2} q^{2}}{4}\right)\right],
$$

where $I_{\nu}$ is a modified Bessel function.

In Figure 3.3 we plot $\widehat{G}_{2 n}(q)$ as a function of wavenumber $q$ for $n=0,1,2$. Note that $\widehat{G}_{2 n}(q)$ alternates in sign with $n$, having a maximum for even $n$ and a minimum for odd $n$. It follows that if $H_{n} \Psi_{2 n}>0$, then the homogeneous state destabilizes 


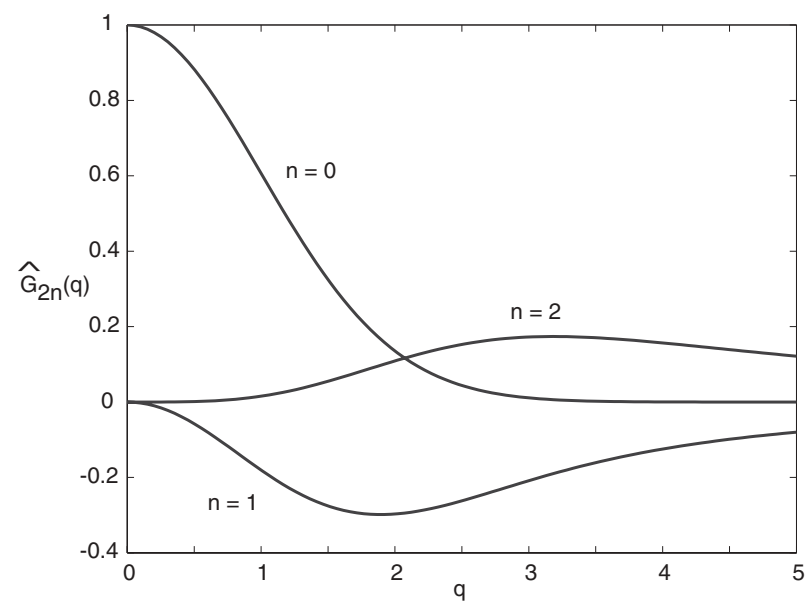

FiG. 3.3. Plot of $\widehat{G}_{2 n}(q)$ as a function of wavenumber $q$ for $n=0,1,2$.
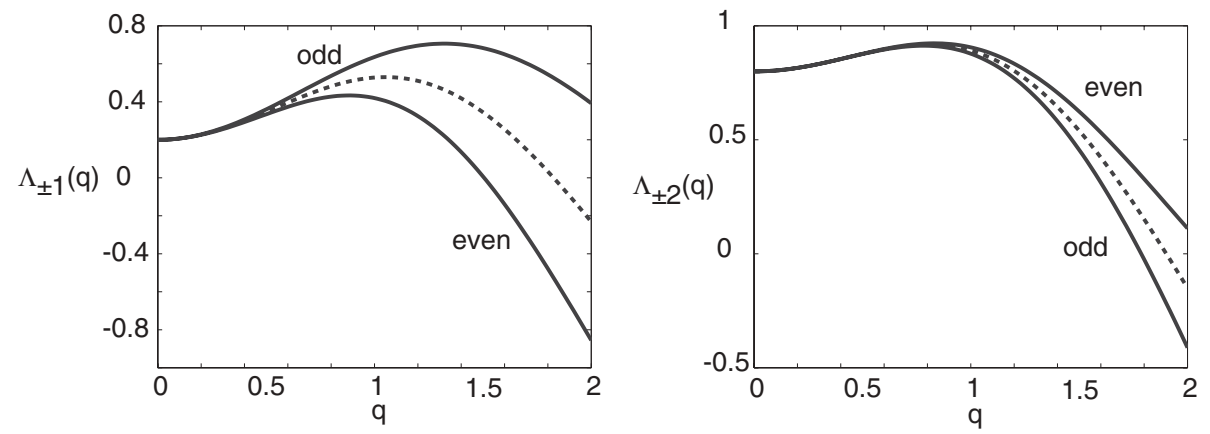

FIG. 3.4. Plot of $\Lambda_{ \pm n}(q)$ as a function of wavenumber $q$ for $n=1,2$. Here $D_{2}=0.2, \eta_{1}=$ $0.3, \eta_{2}=1, H_{n}=1$, and $\beta \Psi_{2 n}=0.5$. The dashed curve represents the coalescence of the even and odd branches when $\beta=0$.

due to excitation of even (odd) eigenmodes when $n$ is even (odd); the opposite result holds for $H_{n} \Psi_{2 n}<0$. Example dispersion curves $\Lambda_{ \pm n}(q)$ are plotted in Figure 3.4 for $n=1,2$. Since $\max _{q}\left\{\Lambda_{-1}(q)\right\}>\max _{q}\left\{\Lambda_{1}(q), \Lambda_{ \pm 2}(q) / 4\right\}>0$ for the given parameter values, it follows that the critical eigenmodes are odd patterns with $n=1$ (assuming $D_{2}>D_{2,0}^{\prime}$ ). Keeping the same parameters but setting $H_{1}=0$ would change the critical eigenmodes to even patterns with $n=2$. For this particular example, the splitting of the even and odd modes around the critical point is small.

4. Doubly periodic patterns. Rotation symmetry implies that in the case of nonzero critical wavenumber $q_{c}$, the space of marginally stable eigenfunctions is infinite-dimensional, consisting of all solutions of the form $u(\theta-\varphi) \mathrm{e}^{i \mathbf{k}_{\varphi} \cdot \mathbf{r}}$, where $u(\theta)$ is either an even or odd function of $\theta, \mathbf{k}_{\varphi}=q_{c}(\cos \varphi, \sin \varphi)$, and $0 \leq \varphi<2 \pi$. However, translation symmetry allows us to restrict the space of solutions of the original equation (2.7) to that of doubly periodic functions. This restriction is standard in many treatments of spontaneous pattern formation, but as yet it has no formal justification. However, there is a wealth of evidence from experiments on convecting fluids and chemical reaction-diffusion systems [23] indicating that such systems tend to generate 
TABLE 4.1

Generators for the planar lattices and their dual lattices.

\begin{tabular}{|c|cc|cc|}
\hline Lattice & $\boldsymbol{\ell}_{1}$ & $\boldsymbol{\ell}_{2}$ & $\hat{\boldsymbol{\ell}}_{1}$ & $\hat{\boldsymbol{\ell}}_{2}$ \\
\hline Square & $(1,0)$ & $(0,1)$ & $(1,0)$ & $(0,1)$ \\
Hexagonal & $\left(1, \frac{1}{\sqrt{3}}\right)$ & $\left(0, \frac{2}{\sqrt{3}}\right)$ & $(1,0)$ & $\frac{1}{2}(-1, \sqrt{3})$ \\
Rhombic & $(1,-\cot \eta)$ & $(0, \csc \eta)$ & $(1,0)$ & $(\cos \eta, \sin \eta)$ \\
\hline
\end{tabular}

doubly periodic patterns in the plane when the homogeneous state is destabilized. Given such a restriction, the associated space of marginally stable eigenfunctions is finite-dimensional. A finite set of specific eigenfunctions can then be identified as candidate planforms, in the sense that they approximate time-independent solutions of (2.7) sufficiently close to the critical point where the homogeneous state loses stability.

Let $\mathcal{L}$ be a planar lattice; that is, choose two linearly independent vectors $\boldsymbol{\ell}_{1}$ and $\ell_{2}$ and let

$$
\mathcal{L}=\left\{2 \pi d\left(m_{1} \ell_{1}+m_{2} \ell_{2}\right): m_{1}, m_{2} \in \mathbf{Z}\right\}
$$

where $d$ is the lattice spacing. Note that $\mathcal{L}$ is a subgroup of the group of planar translations. A function $f: \mathbf{R}^{2} \times \mathbf{S}^{1} \rightarrow \mathbf{R}$ is doubly periodic with respect to $\mathcal{L}$ if

$$
f(\mathbf{r}+\ell, \theta)=f(\mathbf{r}, \theta)
$$

for every $\ell \in \mathcal{L}$. Let $\theta$ be the angle between the two basis vectors $\boldsymbol{\ell}_{1}$ and $\boldsymbol{\ell}_{2}$. We can then distinguish three types of lattice according to the value of $\theta$ : square $(\theta=\pi / 2)$, rhombic $(0<\theta<\pi / 2, \theta \neq \pi / 3)$, and hexagonal $(\theta=\pi / 3)$. After rotation, the generators of the planar lattices are given in Table 4.1 (for unit lattice spacing). Also shown are the generators of the dual lattice

$$
\hat{\mathcal{L}}=\left\{d^{-1}\left(m_{1} \hat{\ell}_{1}+m_{2} \hat{\ell}_{2}\right): m_{1}, m_{2} \in \mathbf{Z}\right\}
$$

with $\boldsymbol{\ell}_{i} \cdot \hat{\ell}_{j}=\delta_{i, j}$. Restriction to double periodicity means that the original Euclidean symmetry group is now restricted to the symmetry group of the lattice, $\Gamma=H_{\mathcal{L}} \dot{+} \mathbf{T}^{2}$, where $H_{\mathcal{L}}$ is the holohedry of the lattice, the subgroup of $\mathbf{O}(2)$ that preserves the lattice, and $\mathbf{T}^{2}$ is the two torus of planar translations modulo the lattice. Thus, the holohedry of the rhombic lattice is $\mathbf{D}_{2}$, the holohedry of the square lattice is $\mathbf{D}_{4}$, and the holohedry of the hexagonal lattice is $\mathbf{D}_{6}$.

Imposing double periodicity on the marginally stable eigenfunctions means restricting the lattice spacing $d$ so that the critical wavevector $\mathbf{k}$ lies on the dual lattice. There are infinitely many choices for the lattice size that satisfy this constraintwe select the one for which $q_{c}$ is the shortest length of a dual wavevector, that is, $q_{c}=d^{-1}$. Linear combinations of eigenfunctions that generate doubly periodic solutions corresponding to dual wavevectors of shortest length are given by

$$
a(\mathbf{r}, \theta)=\sum_{j=1}^{N} z_{j} u\left(\theta-\varphi_{j}\right) \mathrm{e}^{i \mathbf{k}_{j} \cdot \mathbf{r}}+\text { c.c. }
$$

where the $z_{j}$ are complex amplitudes. Here $N=2$ for the square lattice with $\mathbf{k}_{1}=\mathbf{k}_{c}$ and $\mathbf{k}_{2}=R_{\pi / 2} \mathbf{k}_{c}$, where $R_{\xi}$ denotes rotation through an angle $\xi$. Similarly, $N=3$ for the hexagonal lattice with $\mathbf{k}_{1}=\mathbf{k}_{c}, \mathbf{k}_{2}=R_{2 \pi / 3} \mathbf{k}_{c}$, and $\mathbf{k}_{3}=R_{4 \pi / 3} \mathbf{k}_{c}=-\mathbf{k}_{1}-\mathbf{k}_{2}$. It follows that the space of marginally stable eigenfunctions can be identified with 
the $N$-dimensional complex vector space spanned by the vectors $\left(z_{1}, \ldots, z_{N}\right) \in \mathbf{C}^{N}$, with $N=2$ for square or rhombic lattices and $N=3$ for hexagonal lattices. It can be shown that these form $\Gamma$-irreducible representations. The actions of the group $\Gamma$ on $\mathbf{C}^{N}$ can then be explicitly written down for both the odd and even cases [3, 4]. For example, on a hexagonal lattice, a translation $(\mathbf{r}, \theta) \rightarrow(\mathbf{r}+\mathbf{s}, \theta)$ induces the action

$$
\gamma \circ\left(z_{1}, z_{2}, z_{3}\right)=\left(z_{1} \mathrm{e}^{-i \xi_{1}}, z_{2} \mathrm{e}^{-i \xi_{2}}, z_{3} \mathrm{e}^{i\left(\xi_{1}+\xi_{2}\right)}\right),
$$

where $\xi_{j}=\mathbf{k}_{j} \cdot \mathbf{s}$, a rotation $(\mathbf{r}, \theta) \rightarrow\left(R_{2 \pi / 3} \mathbf{r}, \theta+2 \pi / 3\right)$ induces the action

$$
\gamma \circ\left(z_{1}, z_{2}, z_{3}\right)=\left(z_{3}, z_{1}, z_{2}\right)
$$

and a reflection $\kappa$ across the $x$-axis (assuming $\mathbf{k}_{c}=q_{c}(1,0)$ ) induces the action

$$
\gamma \circ\left(z_{1}, z_{2}, z_{3}\right)=\left(z_{1}, z_{3}, z_{2}\right)
$$

The next important observation is that, using weakly nonlinear analysis and perturbation methods, it is possible to reduce the infinite-dimensional system (2.7) to a finite set of coupled ODEs constituting an amplitude equation for $\mathbf{z}$,

$$
\frac{d z_{j}}{d t}=F_{j}(\mathbf{z}), \quad j=1, \ldots, N,
$$

which is equivariant with respect to the induced shift-twist action of the group $\Gamma$ on $\mathbf{C}^{N}$. One can now use techniques from symmetric bifurcation theory to determine the equilibrium solutions that are likely to bifurcate from the homogeneous fixed point $\mathbf{z}=0$. This analysis has been carried out elsewhere within the context of a continuum model of visual cortex [3, 4]. Since the population model (2.7) has the same Euclidean shift-twist symmetry as the cortical model, it has the same restrictions regarding the types of bifurcations from a homogeneous state that can occur. However, which particular bifurcation scenario is realized in practice may differ in the two models. That is, although symmetry considerations restrict the form of the nonlinear functions $F_{j}$ appearing in the amplitude equation $(4.5)[3,4]$, the values of the coefficients multiplying terms at a particular order in $z_{j}$ will be model-dependent. Determining these coefficients would require carrying out an explicit perturbation calculation. Here we focus on general aspects of the bifurcating solutions that can be deduced from symmetry principles. For completeness we briefly review a few basic definitions and results from equivariant bifurcation theory [13].

Isotropy subgroups. The symmetries of any particular equilibrium solution $\mathbf{z}$ form a subgroup called the isotropy subgroup of $\mathbf{z}$ defined by

$$
\Sigma_{\mathbf{z}}=\{\sigma \in \Gamma: \sigma \mathbf{z}=\mathbf{z}\} .
$$

More generally, we say that $\Sigma$ is an isotropy subgroup of $\Gamma$ if $\Sigma=\Sigma_{\mathbf{z}}$ for some $\mathbf{z} \in V$. Isotropy subgroups are defined up to some conjugacy. A group $\Sigma$ is conjugate to a group $\widehat{\Sigma}$ if there exists $\sigma \in \Gamma$ such that $\widehat{\Sigma}=\sigma^{-1} \Sigma \sigma$. The fixed-point subspace of an isotropy subgroup $\Sigma$, denoted by $\operatorname{Fix}(\Sigma)$, is the set of points $\mathbf{z} \in V$ that are invariant under the action of $\Sigma$,

$$
\operatorname{Fix}(\Sigma)=\{\mathbf{z} \in V: \sigma \mathbf{z}=\mathbf{z} \forall \sigma \in \Sigma\} .
$$

Finally, the group orbit through a point $\mathbf{z}$ is

$$
\Gamma \mathbf{z}=\{\sigma \mathbf{z}: \sigma \in \Gamma\} .
$$


TABLE 4.2

Even planforms with $u(-\theta)=u(\theta)$. The hexagon solutions $(0)$ and $(\pi)$ have the same isotropy subgroup, but they are not conjugate solutions.

\begin{tabular}{|c|c|c|}
\hline Lattice & Name & Planform eigenfunction \\
\hline Square & Even square & $u(\theta) \cos x+u\left(\theta-\frac{\pi}{2}\right) \cos y$ \\
& Even roll & $u(\theta) \cos x$ \\
\hline Rhombic & Even rhombic & $u(\theta) \cos \left(\mathbf{k}_{1} \cdot \mathbf{r}\right)+u(\theta-\eta) \cos \left(\mathbf{k}_{2} \cdot \mathbf{r}\right)$ \\
& Even roll & $u(\theta) \cos \left(\mathbf{k}_{1} \cdot \mathbf{r}\right)$ \\
\hline Hexagonal & Even hexagon $(0)$ & $u(\theta) \cos \left(\mathbf{k}_{1} \cdot \mathbf{r}\right)+u\left(\theta+\frac{\pi}{3}\right) \cos \left(\mathbf{k}_{2} \cdot \mathbf{r}\right)+u\left(\theta-\frac{\pi}{3}\right) \cos \left(\mathbf{k}_{3} \cdot \mathbf{r}\right)$ \\
& Even hexagon $(\pi)$ & $u(\theta) \cos \left(\mathbf{k}_{1} \cdot \mathbf{r}\right)+u\left(\theta+\frac{\pi}{3}\right) \cos \left(\mathbf{k}_{2} \cdot \mathbf{r}\right)-u\left(\theta-\frac{\pi}{3}\right) \cos \left(\mathbf{k}_{3} \cdot \mathbf{r}\right)$ \\
& Even roll & $u(\theta) \cos \left(\mathbf{k}_{1} \cdot \mathbf{r}\right)$ \\
\hline
\end{tabular}

If $\mathbf{z}$ is an equilibrium solution of (4.5), then so are all other points of the group orbit (by equivariance). One can now adopt a strategy that restricts the search for solutions of (4.5) to those that are fixed points of a particular isotropy subgroup. In general, if a dynamical system is equivariant under some symmetry group $\Gamma$ and has a solution that is a fixed point of the full symmetry group, then we expect a loss of stability to occur upon variation of one or more system parameters. Typically such a loss of stability will be associated with the occurrence of new solution branches with isotropy subgroups $\Sigma$ smaller than $\Gamma$. One says that the solution has spontaneously broken symmetry from $\Gamma$ to $\Sigma$. Instead of a unique solution with the full set of symmetries $\Gamma$, a set of symmetrically related solutions (orbits under $\Gamma$ modulo $\Sigma$ ) each with symmetry group (conjugate to) $\Sigma$ is observed.

Equivariant branching lemma (see [13]). The system of equations (4.5) has a fixed point $z=0$ of the full symmetry group $\Gamma$. The equivariant branching lemma states that generically there exists a (unique) equilibrium solution bifurcating from the fixed point for each of the axial subgroups of $\Gamma$ under the given group action - a subgroup $\Sigma \subset \Gamma$ is axial if $\operatorname{dim} \operatorname{Fix}(\Sigma)=1$. The heuristic idea underlying this lemma is as follows. Let $\Sigma$ be an axial subgroup and $\mathbf{z} \in \operatorname{Fix}(\Sigma)$. Equivariance of $F$ then implies that

$$
\sigma F(\mathbf{z})=F(\sigma \mathbf{z})=F(\mathbf{z})
$$

for all $\sigma \in \Sigma$. Thus $F(\mathbf{z}) \in \operatorname{Fix}(\Sigma)$ and the system of coupled ODEs (4.5) can be reduced to a single equation in the fixed-point space of $\Sigma$. Thus one can systematically identify the various expected primary bifurcation branches by constructing the associated axial subgroups and finding their fixed points. The calculation of these subgroups has been carried out elsewhere [3, 4], and the resulting even and odd planforms are listed in Tables 4.2 and 4.3 .

One way to represent the planforms graphically is to indicate the direction(s) of preferred orientation at each point in space $\mathbf{r}$, that is, the orientations that maximize the state $a(\mathbf{r}, \theta)$ for fixed $\mathbf{r}$. This has been carried out elsewhere in the case of cortical patterns, where the preferred orientation corresponds to the orientation of a local visual stimulus that elicits the maximum response of a neuron at a particular location in the cortex $[3,4]$. From a mathematical rather than a physical viewpoint, the only difference between the cortical patterns and those of the population model is that in the former case the functions $u(\theta)$ are always restricted to be $\pi$-periodic, and hence, the resulting spatio-angular patterns are line fields. As a simple example, consider a square lattice with $u(\theta)=\cos 2 \theta$ or $u(\theta)=\sin 2 \theta$ and $\mathbf{k}_{c}=2 \pi(1,0)$. The 
TABLE 4.3

Odd planforms with $u(-\theta)=-u(\theta)$.

\begin{tabular}{|c|c|c|}
\hline Lattice & Name & Planform eigenfunction \\
\hline Square & Odd square & $u(\theta) \cos x-u\left(\theta-\frac{\pi}{2}\right) \cos y$ \\
& Odd roll & $u(\theta) \cos x$ \\
\hline Rhombic & Odd rhombic & $u(\theta) \cos \left(\mathbf{k}_{1} \cdot \mathbf{r}\right)+u(\theta-\eta) \cos \left(\mathbf{k}_{2} \cdot \mathbf{r}\right)$ \\
& Odd Roll & $u(\theta) \cos \left(\mathbf{k}_{1} \cdot \mathbf{r}\right)$ \\
\hline Hexagonal & Odd hexagon & $u(\theta) \cos \left(\mathbf{k}_{1} \cdot \mathbf{r}\right)+u\left(\theta+\frac{\pi}{3}\right) \cos \left(\mathbf{k}_{2} \cdot \mathbf{r}\right)+u\left(\theta-\frac{\pi}{3}\right) \cos \left(\mathbf{k}_{3} \cdot \mathbf{r}\right)$ \\
& Triangle & $u(\theta) \sin \left(\mathbf{k}_{1} \cdot \mathbf{r}\right)+u\left(\theta+\frac{\pi}{3}\right) \sin \left(\mathbf{k}_{2} \cdot \mathbf{r}\right)+u\left(\theta-\frac{\pi}{3}\right) \sin \left(\mathbf{k}_{3} \cdot \mathbf{r}\right)$ \\
& Patchwork quilt & $u\left(\theta+\frac{\pi}{3}\right) \cos \left(\mathbf{k}_{2} \cdot \mathbf{r}\right)-u\left(\theta-\frac{\pi}{3}\right) \cos \left(\mathbf{k}_{3} \cdot \mathbf{r}\right)$ \\
& Odd roll & $u(\theta) \cos \left(\mathbf{k}_{1} \cdot \mathbf{r}\right)$ \\
\hline
\end{tabular}
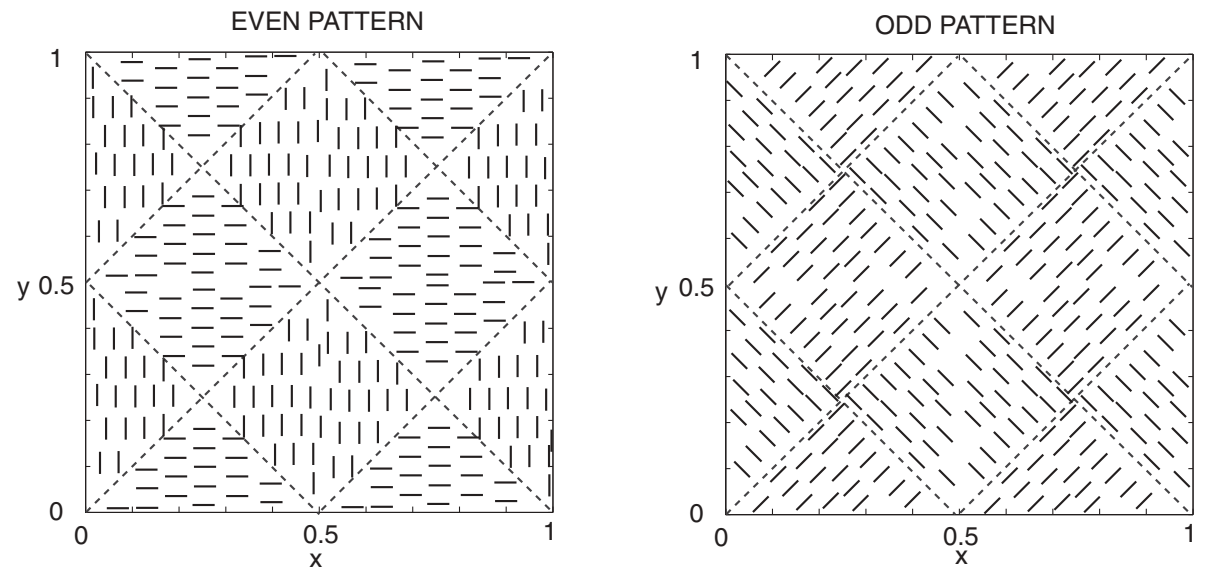

FIG. 4.1. Line fields for even patterns $(u(\theta)=\cos 2 \theta)$ and odd patterns $(u(\theta)=\sin 2 \theta)$ in a fundamental domain of a square lattice. The domain is divided up into subregions where the preferred orientation $(\bmod \pi)$ is uniform. The direction of orientation within each subregion is indicated by the small parallel bars, which could be interpreted as aligned objects at discretely sampled points within the subregion. Dashed lines indicate line singularities separating regions of different orientation.

corresponding even and odd planforms (modulo an arbitrary translation) are

$$
\begin{aligned}
& a_{+}(\mathbf{r}, \theta)=\cos 2 \theta(\sin 2 \pi x-\sin 2 \pi y), \\
& a_{-}(\mathbf{r}, \theta)=\sin 2 \theta(\sin 2 \pi x-\sin 2 \pi y) .
\end{aligned}
$$

In the case of the even eigenmode $a_{+}(\mathbf{r}, \theta)$, the preferred orientation $(\bmod \pi)$ at $(x, y)$ is $\theta=0$ when $\sin 2 \pi x>\sin 2 \pi y$ and $\theta=\pi / 2$ when $\sin 2 \pi x<\sin 2 \pi y$. The corresponding preferred orientations of the odd eigenmode $a_{-}(\mathbf{r}, \theta)$ are $\theta=\pi / 4,3 \pi / 4$. Note that line singularities occur for $\sin 2 \pi x=\sin 2 \pi y$, across which there are jumps in orientation preference. The resulting even and odd line fields are shown in Figure 4.1. Inclusion of higher harmonic contributions to the function $u(\theta)$ can lead to point rather than line singularities as well as sites containing more than one preferred orientation [4]. If there is no distinction between "head" and "tail," then solutions of the population model (2.7) will also be $\pi$-periodic. On the other hand, if there is such a distinction, then generically the resulting patterns will be represented by vector fields rather than line fields. In the case of a square lattice with $u(\theta)=\cos \theta$ or $u(\theta)=\sin \theta$ and $\mathbf{k}_{c}=2 \pi(1,0)$, the corresponding even and odd planforms (modulo 

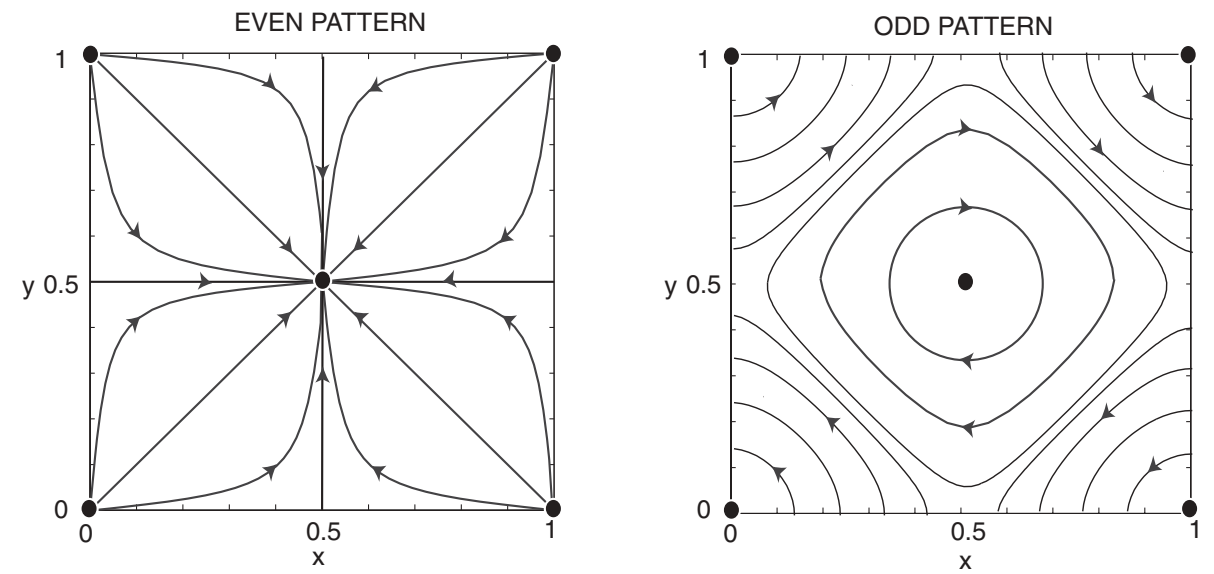

FIG. 4.2. Vector fields for even patterns $(u(\theta)=\cos \theta)$ and odd patterns $(u(\theta)=\sin \theta)$ in a fundamental domain of a square lattice. The preferred orientation at a given point in the plane is given by the direction tangential to the flow line passing through that point. There is no preferred orientation at the singularities (indicated by filled circles).

an arbitrary translation) are

$$
\begin{aligned}
& a_{+}(\mathbf{r}, \theta)=\cos \theta \sin 2 \pi x+\sin \theta \sin 2 \pi y=A(\mathbf{r}) \cos \left[\theta-\theta_{+}(\mathbf{r})\right], \\
& a_{-}(\mathbf{r}, \theta)=\sin \theta \sin 2 \pi x-\cos \theta \sin 2 \pi y=A(\mathbf{r}) \cos \left[\theta-\theta_{-}(\mathbf{r})\right],
\end{aligned}
$$

with $A(\mathbf{r})=\sqrt{\sin ^{2} 2 \pi x+\sin ^{2} 2 \pi y}$ and

$$
\theta_{+}(\mathbf{r})=\tan ^{-1} \frac{\sin 2 \pi y}{\sin 2 \pi x}, \quad \theta_{-}(\mathbf{r})=\tan ^{-1} \frac{-\sin 2 \pi x}{\sin 2 \pi y} .
$$

It follows that the preferred orientation at position $\mathbf{r}$ is $\theta(\mathbf{r})$, provided that $A(\mathbf{r}) \neq 0$; otherwise there is no preferred orientation. Another way to state this is that the preferred orientation is determined by the flow lines of the vector fields,

$$
\mathbf{V}_{+}=\sin 2 \pi x \frac{\partial}{\partial x}+\sin 2 \pi y \frac{\partial}{\partial y}, \quad \mathbf{V}_{-}=-\sin 2 \pi y \frac{\partial}{\partial x}+\sin 2 \pi x \frac{\partial}{\partial y},
$$

except at the singularities $x=m \pi, y=m^{\prime} \pi$ for integers $m, m^{\prime}$. The resulting vector fields are shown in Figure 4.2. Note that if higher harmonics are included in the function $u(\theta)$, then it is possible for the flow lines to intersect, indicating that there can be more than one preferred orientation away from singularities.

5. Discussion. In this paper we have shown that a wide class of self-organizing biological systems have interactions that are invariant with respect to the shift-twist action of the Euclidean group, and that this has major implications for the types of patterns that can arise in these systems. Our main prediction is that patterns with spatio-angular order should exhibit correlations between the directions of preferred orientation and the underlying spatial orientation of the pattern (as determined by the wavevectors of the excited eigenmodes), and that there are two distinct types of correlation corresponding to scalar and pseudoscalar representations of the Euclidean group. In other words, given a spatially periodic variation in preferred orientation, 
there is a correlation between the preferred orientation within a patch and the orientation of the boundaries of that patch. Whether or not such correlations are actually observed in real systems remains to be seen, although the line fields shown in Figure 4.1 are very suggestive of certain arrangements of fibroblasts where the cells appear to be oriented at approximately $45^{\circ}$ to line singularities [18].

One of the simplifying assumptions in our analysis has been to restrict the spatial domain to be two-dimensional. This is appropriate for cells grown in vitro on a flat surface and for animal herds. On the other hand, cells in vivo and fish schools or bird flocks [20] are better described by a three-dimensional domain. In the threedimensional case, the orientation of an individual is specified by points on a sphere $(\phi, \theta)$ with $\phi \in[0, \pi]$ and $\theta \in[0,2 \pi)$. This suggests that the underlying symmetry group is $\mathbf{E}(3) \times \mathbf{O}(3)$ when the corresponding interaction kernel is separable with respect to spatial and angular coordinates. An interesting problem that follows from this is to determine the appropriate three-dimensional analogue of Euclidean shifttwist symmetry when the kernel is taken to be nonseparable. Another factor that would modify the symmetry group is the presence of an environmental gradient that biases the selection of a direction with which to align. Examples include migrating birds using the earth's magnetic field as a directional cue and fibroblasts aligning strongly with grooves on an artificial substrate.

\section{REFERENCES}

[1] I. B. Vivancos, P. Chossat, And I. Melbourne, New planforms in systems of partial differential equations with Euclidean symmetry, Arch. Ration. Mech. Anal., 131 (1995), pp. 199-224.

[2] W. H. Bosking, Y. Zhang, B. Schofield, and D. Fitzpatrick, Orientation selectivity and the arrangement of horizontal connections in tree shrew striate cortex, J. Neurosci., 17 (1997), pp. 2112-2127.

[3] P. C. Bressloff, J. D. Cowan, M. Golubitsky, P. J. Thomas, and M. Wiener, Geometric visual hallucinations, Euclidean symmetry and the functional architecture of striate cortex, Phil. Trans. Roy. Soc. London B, 356 (2001), pp. 299-330.

[4] P. C. Bressloff, J. D. Cowan, M. Golubitsky, and P. J. Thomas, Scalar and pseudoscalar bifurcations: Pattern formation on the visual cortex, Nonlinearity, 14 (2001), pp. 739-775.

[5] D. Chilingworth And M. Golubitsky, Symmetry and pattern formation in a planar layer of nematic liquid crystal, J. Math. Phys., 44 (2003), pp. 4201-4219.

[6] J. CooK, Waves of alignment in populations of interacting, oriented individuals, Forma, 10 (1995), pp. 171-203.

[7] G. Civelekoglu and L. Edelstein-Keshet, Modelling the dynamics of F-actin in the cell, Bull. Math. Biol., 56 (1994), pp. 587-616.

[8] J. C. Dallon and J. A. Sherratt, A mathematical model for spatially varying extracellular matrix alignment, SIAM J. Appl. Math., 61 (2000), pp. 506-527.

[9] P. De Gennes, The Physics of Liquid Crystals, Clarendon Press, Oxford, UK, 1974.

[10] T. R. Elsdale And J. B. L. Bard, Collagen substrata for studies on cell behavior, J. Cell Biol., 54 (1972), pp. 626-537.

[11] G. B. Ermentrout and J. D. Cowan, A mathematical theory of visual hallucination patterns, Biol. Cybernetics, 34 (1979), pp. 137-150.

[12] E. Geigant, K. Ladizhansky, and A. Mogilner, An integrodifferential model for orientation distributions of f-actin in cells, SIAM J. Appl. Math., 59 (1998), pp. 787-809.

[13] M. Golubitsky and I. Stewart, The Symmetry Perspective: From Equilibrium to Chaos in Phase Space and Physical Space, Birkhäuser, Basel, 2002.

[14] D. Grunbaum and A. Okubo, Modelling social animal aggregations, in Frontiers of Theoretical Biology, Lecture Notes in Biomath. 100, S. A. Levin, ed., Springer-Verlag, Berlin, 1994, pp. 296-325.

[15] C. T. Lee, M. F. Hoopes, J. Diehl, W. Gilliland, G. Huxel, E. V. Leaver, K. McCann, J. Umbanhowar, and A. Mogilner, Non-local concepts and models in biology, J. Theoret. Biol., 210 (2001), pp. 201-219. 
[16] A. Mogilner And L. Edelstein-Keshet, Selecting a common direction I: How orientational order can arise from simple contact responses between interacting cells, J. Math. Biol., 33 (1995), pp. 619-660.

[17] A. Mogilner, L. Edelstein-Keshet, And G. B. Ermentrout, Selecting a common direction II: Peak-like solutions representing total alignment of cell clusters, J. Math. Biol., 34 (1996), pp. 811-842.

[18] A. Mogilner and L. Edelstein-Keshet, Spatio-angular order in populations of self-aligning objects: Formation of oriented patches, Phys. D, 89 (1996), pp. 346-367.

[19] A. Okubo, Dynamical aspects of animal grouping: Swarms, schools, flocks and herds, Adv. Biophys., 22 (1986), pp. 1-94.

[20] J. Parrish and W. Hammner, eds., 3D Animal Aggregations, Cambridge University Press, Cambridge, UK, 1994.

[21] J. A. Sherratt And M. E. Lewis, Stress-induced alignment of actin filaments and the mechanics of cytogel, Bull. Math. Biol., 55 (1993), pp. 637-654.

[22] T. P. Stossel, How cells crawl, Amer. Sci., 78 (1990), pp. 408-423.

[23] D. Walgraef, Spatio-Temporal Pattern Formation, Springer-Verlag, Berlin, 1997.

[24] L. R. Williams And D. W. Jacobs, Stochastic completion fields: A neural model of illusory contour shape and salience, Neural Comput., 9 (1999), pp. 849-870. 